\title{
Obesidade: inflamação e compostos bioativos
}

\section{Obesity: Inflammation and Bioactive Compounds}

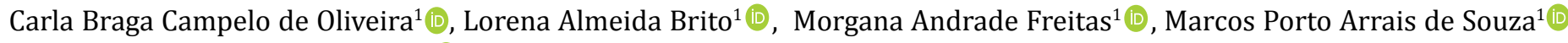 \\ Juliana Magalhães da Cunha Rêgo ${ }^{2}$ (D), Richele Janaina de Araújo Machado ${ }^{2}$ (i)
}

1. Graduação em Nutrição pelo Centro Universitário Christus (Unichristus), Fortaleza, CE, Brasil. 2. Docente do curso de graduação em Nutrição pelo Centro Universitário Christus (Unichristus), Fortaleza, CE, Brasil.

\begin{abstract}
Resumo
Objetivo: realizar um levantamento bibliográfico com enfoque nos compostos bioativos que modulam a inflamação na obesidade. Métodos: foram utilizadas as bases de dados Scientific Eletronic Library Online e PubMed, com artigos de revisão e originais, em língua portuguesa e inglesa, publicados entre 2010 e 2019, adotando os seguintes descritores: obesity, inflammation e bioactive compounds. Resultados: alimentos com propriedades funcionais e fontes de compostos bioativos podem auxiliar na modulação de citocinas pró-inflamatórias, como a curcumina no açafrão da terra, as catecolaminas no chá-verde, punicalagina na romã, a $\beta$-glucana na aveia e os polifenóis no azeite de oliva. Tais compostos geralmente atuam inibindo mediadores, como fator nuclear Kappa $\beta$, diminuindo marcadores inflamatórios, como fator de necrose tumoral $\alpha$ e as interleucinas $(1,2,6$, 8, e 12). Conclusão: tendo em vista o potencial dos alimentos em modular a inflamação na obesidade, o conhecimento de suas propriedades funcionais é importante por auxiliar os profissionais de Nutrição no planejamento de estratégias para a prevenção e o tratamento da obesidade.
\end{abstract}

Palavras-chave: Doenças crônicas. Alimento funcional. Compostos Fitoquímicos.

\begin{abstract}
Obijective: To perform a bibliographical survey with a focus on bio-active compounds with the potential to modulate inflammation in obesity. Methods: Scientific Electronic Library Online and PubMed databases were used, with review articles and originals, in Portuguese and English, published between 2010 and 2019, adopting the following descriptors: obesity, inflammation e bioactive compounds. Results: Foods with functional properties, sources of bioactive compounds may aid in the modulation of pro-inflammatory cytokines such as curcumin in earth turmeric, catecholamines in green tea, punicalagin in pomegranate, $\beta$-glucan in oats and polyphenols in olive oil which mostly act to inhibit mediators such as nuclear factor Kappa $\beta$, thereby decreasing inflammatory markers such as tumor necrosis factor $\alpha$ and interleukins $1,2,6,8$, and 12 . Conclusion: Considering the potential of food to modulate inflammation in obesity, knowledge about its functional properties is important as it assists nutrition professionals in planning strategies for obesity prevention and treatment.
\end{abstract}

Keywords: Chronic diseases. Functional food. Phytochemicals.

\section{INTRODUÇÃO}

A obesidade é um estado patológico resultante do acúmulo de excesso de gordura corporal, principalmente na região abdominal, que ocorre devido ao desequilíbrio entre a ingestão de energia e seu gasto, levando o indivíduo a desenvolver excesso de tecido adiposo ao ponto de impactar negativamente na sua saúde ${ }^{1}$.

No Brasil, segundo dados da pesquisa Vigilância de Fatores de Risco e Proteção para Doenças Crônicas por Inquérito Telefônico (VIGITEL), realizada em todas as capitais brasileiras, com 53,2 mil pessoas acima de 18 anos, em 2006, a prevalência da obesidade era de $11,8 \%$ e aumentou para $18,9 \%$ em 2016. Essa doença pode trazer consequências negativas para a saúde dos indivíduos, por meio das doenças crônicas não transmissíveis (DCNTs), como diabetes mellitus tipo 2, a hipertensão arterial e as dislipidemias, que têm como uma das causas o excesso de gordura corporal ${ }^{2}$.
Em indivíduos obesos, o tecido adiposo eleva a síntese de adipocinas (citocinas), modifica a microbiota intestinal e aumenta as espécies reativas de oxigênio, que geram um estresse oxidativo e, consequentemente, um dano celular ou tecidual, favorecendo o desenvolvimento das DCNTs ${ }^{3,4}$.

As mudanças na alimentação, com a inserção de alimentos contendo componentes que podem favorecer um melhor funcionamento do organismo, como os compostos bioativos (polifenóis, ácidos fenólicos ou flavonoides), contribuem para a prevenção e a modulação das vias metabólicas associadas ao desenvolvimento das DCNTs, entre elas, a obesidade ${ }^{5}$.

Considerando a obesidade um problema de saúde pública, este trabalho teve como objetivo realizar um levantamento bibliográfico com enfoque nos compostos bioativos que modulam a inflamação na obesidade. 


\section{MÉTODOS}

A pesquisa bibliográfica foi realizada por meio de consulta à base de dados Scientific Eletronic Library Online (SciELO) e PubMed. Foram utilizados artigos de revisão e originais, em língua portuguesa e inglesa, publicados entre 2010 e 2019, após a análise do título, resumo e textos completos. Utilizaramse os seguintes descritores: obesity, inflammation e bioactive compounds e todas as combinações de associação entre elas.

\section{RESULTADOS}

Obesidade: epidemiologia, definição, fatores de risco e fisiopatologia

De acordo com a pesquisa do Ministério da Saúde, o número de obesos no país aumentou $67,8 \%$ nos últimos treze anos, pois, em 2006, 11,8\% da população era obesa, aumentando, assim, para $19,8 \%$ em 2018. Esses dados também apontam que $84,2 \%$ dos adultos que possuem idade entre 25 a 34 anos são obesos, e, na faixa etária de 35 a 44 anos, esse percentual é de $81,1 \%$. Destaca-se, também, que há um aumento do percentual em mulheres $(20,7 \%)$ em relação aos homens $(18,7 \%)$. A VIGITEL Proteção para DCNTs registrou um aumento no número de brasileiros com excesso de peso, representando, assim, 55,7\% da população².

Evidenciada, atualmente, como um dos maiores problemas de saúde pública do mundo, a obesidade é uma condição crônica multifatorial complexa, que possui relação à manifestação de insegurança alimentar e nutricional por meio de fatores condicionantes, como alimentação rica em gorduras e açúcares e o consumo excessivo de alimentos ultraprocessados, associados ao sedentarismo ${ }^{6}$.

Existem diversos mecanismos fisiopatológicos na obesidade que participam da regulação do apetite e da ingestão alimentar, do aumento do tecido adiposo e da consequente resistência à insulina, alterando as funções metabólicas e endócrinas desse tecido ${ }^{7}$. No obeso, há níveis elevados de adipocinas (citocinas) pró-inflamatórias, como o angiotensinogêneo, fator de necrose tumoral (TNF- $\alpha$ ), a interleucina ${ }^{6}$ (IL-6), a leptina, proteína quimioatraente de monócito-1 (MCP-1) e a resistina ${ }^{7,8}$.

A leptina, um hormônio pelo tecido adiposo, é responsável pela regulação da fome, sinalizando a saciedade ao sistema nervoso central (SNC), regulando o armazenamento e o uso de energia pelo corpo em longo prazo para manter seu equilíbrio. Porém, no obeso, há alteração na sinalização da leptina no controle do apetite, devido à resistência a tal hormônio, mesmo em suas quantidades elevadas ${ }^{9}$.

Outro hormônio regulador de apetite é a grelina, responsável por induzir a fome, seu alvo é o núcleo do hipotálamo e o tronco encefálico, fazendo parte do sistema nervoso simpático (SNS) ${ }^{10}$. Ele é sintetizado em maior quantidade no fundo gástrico por glândulas oxínticas, e, em menor quantidade, no corpo do estômago. Em indivíduos obesos, os níveis séricos de grelina são menores se comparados a indivíduos magros, devido à alteração em seu gene ou à presença de anticorpos para seus receptores ${ }^{11}$.

\section{Obesidade e Inflamação}

Existe uma associação entre estilo de vida inadequado e obesidade que modifica a resposta fisiológica pelo aumento dos fatores pró-inflamatórios (adipocitocinas), que são produzidas pelos adipócitos e por meio da destruição das células adiposas, permitindo que a inflamação se torne crônica, alterando seu funcionamento ${ }^{12}$.

O adipócito sofre hipertrofia e expande o tecido adiposo, levando a uma baixa perfusão e à hipóxia celular que, por sua vez, podem iniciar a inflamação, induzindo o programa do gene HIF1 que funciona como um regulador de transcrição da resposta adaptativa ${ }^{13}$.

$\mathrm{O}$ tecido adiposo produz adipocinas anti-inflamatórias, como a interleucina 10 (IL-10) e a adiponectina (AdipoQ), que são capazes de inibir a síntese de adipocinas pró-inflamatórias por linfócitos T e macrófagos. Ela inibe a síntese do TNF- $\alpha$, interleucina 1 (IL-1), IL-6 e interleucina 8 (IL-8) em monócitos ${ }^{8}$. A AdipoQ melhora a sensibilidade à insulina, possui ação antiinflamatória, reduzindo os níveis séricos das adipocinas próinflamatórias IL- 6 e o TNF- $\alpha$, e contribui para a homeostase pós-prandial da glicose e de lipídeos, provocando o aumento da oxidação de gorduras além de propriedade antiaterogênica ${ }^{14}$.

A expressão de citocinas inflamatórias (TNF- $\alpha$ e IL-6) é induzida por quinases do tecido adiposo e hepático. Nos obesos, tem uma expressão aumentada. Essas quinases fazem a regulação de programas de transcrição, como fator nuclear Kappa $\beta$ (NF$\mathrm{k} \beta$ ) e fator regulador do Interferon $\gamma$ (IFN- $\gamma$ ), induzindo o gene mediador inflamatório. $\mathrm{O}$ aumento dessas citocinas eleva a ativação do receptor, estabelecendo um ciclo de feedback positivo da inflamação e de vias metabólicas sinalizadas, tendo o inflamassoma que inicia a resposta inflamatória e receptores Toll-like do sistema imune inato ativados ${ }^{15}$.

Em obesos, ocorre uma resposta do sistema imune, ativada como resposta à inflamação, cujos macrófagos vão aumentando devido a seu recrutamento e exibindo um cenário pró-inflamatório, expressando, assim, citocinas inflamatórias. Esse aumento de macrófagos é devido à inflamação do tecido adiposo que se desloca junto com a obesidade, desenvolvendo resistência à insulina (RI) e à doença metabólica ${ }^{13}$.

$O$ indivíduo obeso apresenta uma maior secreção de adipocinas pró-inflamatórias (TNF- $\alpha$, IL-6, IL-8, IL-1) e menor das antiinflamatórias (IL-10 e AdipoQ), caracterizando a obesidade como uma inflamação crônica que causa grande impacto em diversas funções corporais e ocasiona o desenvolvimento de doenças cardiovasculares (DCV), RI, hipertrigliceridemia e hipercolesterolemia e câncer ${ }^{7,13}$.

Alimentos e compostos bioativos moduladores da inflamação 
Diversos alimentos funcionais contêm componentes que podem melhorar o funcionamento do organismo, uma vez que a diminuição do consumo desses alimentos tem sido considerado um fator de risco para o desenvolvimento das DCNTs ${ }^{16}$.

Existe uma grande variabilidade de verduras, legumes e frutas que apresentam compostos bioativos, como polifenóis, carotenoides, isoflavonas, resveratrol, catequinas e os flavonoides. A ingestão desses compostos deve ser diária, para atender às necessidades e ter ação multifatorial ${ }^{17}$.

Os alimentos funcionais destacados na prevenção e no tratamento da obesidade, e que podem ser utilizados como estratégia nutricional são açafrão (Curcuma longa L.), chá-verde (Camellia sinensis), romã (Punica granatum L.), aveia (Avena sativa L.) e o azeite de oliva (Olea europaea).

O açafrão contém um polifenol (curcumina), que possui características antioangiogênicas, anti-inflamatórias e antiobesidade ${ }^{18}$. A curcumina é o principal composto bioativo do açafrão e apresenta-se como um aliado ao tratamento de várias doenças inflamatórias. Ela atua nas enzimas ciclooxigenase-2 (COX- 2) e lipoxigenase (LOX), integrantes da cascata do ácido araquidônico envolvido na inflamação; sobre a DNA polimerase, e nos fatores de transcrição, como o NF-k $\beta$, bloqueando sua ativação que promove expressão de mediadores inflamatórios (TNF- $\alpha$, IL-1, IL-2, IL-6, IL-8, IL-12) ${ }^{19}$.

A suplementação oral de $1 g$ de curcumina por 30 dias em indivíduos obesos reduziu o estresse oxidativo ${ }^{20}$. A utilização da curcumina (500 mg/dia) associada com a piperina, um composto bioativo presente na pimenta (Piper nigrum), (5mg/dia) por 30 dias, reduziu os níveis séricos de citocinas inflamatórias (IL-1ß e IL-4) em indivíduos obesos ${ }^{21}$. Em um estudo com suplementação oral de curcumina com $1 \mathrm{~g}$ por dia, durante 30 dias, houve eficácia na redução de estresse oxidativo em indivíduos obesos ${ }^{20}$.

O chá-verde contém polifenóis, catequinas e cafeína, que desempenham um papel importante na redução e na manutenção de peso adequado ${ }^{22}$. Tal alimento possui propriedade anti-inflamatória e pode ser usado como estratégia para modular a inflamação e o estresse oxidativo ${ }^{23}$.

Nos polifenóis do chá-verde, um dos mecanismos propostos que se dá pelo controle do apetite, regulação negativa de enzimas, envolvendo metabolismo lipídico hepático e a diminuição da absorção de nutrientes sob a influência do sistema nervoso simpático (SNS). A norepinafrina (NE) estimula a lipólise em tecidos periféricos (adiposo, fígado, músculo esquelético), liberando ácidos graxos livres e, com a regulação positiva do metabolismo lipídico hepático, aumenta a estimulação simpática pela inibição da catecol-o-metiltransferase (COMT) e fosfodiesterase ${ }^{24}$.

Entre as catequinas do chá-verde, o galato de epigalocatequina
(EGCG) é considerado o mais importante devido ao aumento da oxidação dos ácidos graxos. Em um ensaio clínico randomizado, o consumo de chá-verde por oito semanas (928 mg catequinas dos quais $440 \mathrm{mg}$ de EGCG e 8,96 mg de cafeína), diminuiu o peso corporal e o perímetro da cintura em indivíduos com obesidade e síndrome metabólica ${ }^{25}$. A recomendação para o consumo do chá-verde é de uma a duas xícaras $(240 \mathrm{ml}$ a 480 $\mathrm{ml})$ por dia ${ }^{26}$.

A romã (Punica granatum L.) é utilizada para tratar infecções e possui efeitos antiobesidade devido às variedades de compostos bioativos encontrados ${ }^{27}$.

Ela é uma fonte de elagitaninos (ET) punicalagina, o que despertou considerável interesse pela fruta da romã. Estudos mostram efeitos anti-inflamatórios da romã e seus compostos biologicamente ativo na redução do estresse oxidativo pela inibição de NF-K $\beta^{28,29}$. Os compostos bioativos (punicalagina) do suco de romã suprimiram a ativação do NF-k $\beta^{30}$.

A aveia é fonte de fibra dietética solúvel ( $\beta$-glucana) que apresenta efeitos antiobesidade ${ }^{31}$. Os ácidos graxos de cadeia curta (AGCC - acetato, propianto e butirato), produzidos durante a fermentação bacteriana, são substratos para a microbiota intestinal e exercem papel anti-inflamatório no tecido adiposo branco, pela redução na concentração de gordura ${ }^{32}$.

Esses AGCCs são essenciais para as funções imunológicas. No estudo com células intestinais de camundongos, houve uma redução da ativação do NF-k $\beta$ pelo butirato e uma ativação da interleucina-18 (IL-18) que favorece a regulação da inflamação ${ }^{33}$. O consumo de fibra dietética, acima de $10 \mathrm{~g} / 1000 \mathrm{kcal} / \mathrm{dia}$ ou cerca de $20 \mathrm{~g} /$ dia, pode estar relacionado com a perda de peso corporal, variando de 1,2 a 3,6 kg em 8-12 anos ${ }^{34}$.

O azeite de oliva é o óleo extraído da fruta da oliveira (Oleaeuropaea L.). A composição do polifenol do azeite está relacionada com os glicosídeos da fruta. A ingestão do azeite de oliva reduz os marcadores inflamatórios pela inibição do NF-k $\beta^{35}$. Logo, essas propriedades do azeite têm grandes influências nas características dos mediadores antiinflamatórios, reduzindo os níveis de INF- $\gamma$, IL-6, TNF- $\alpha$, COX-2 no plasma, que terá funções antioxidantes, anti-inflamatórias, melhora a sensibilidade à insulina, a proteção cardiovascular, pelo efeito antiaterogênico (com a ingestão de $50 \mathrm{~mL} /$ dia do azeite extravirgem), neuroprotetor, e anticancerígeno ${ }^{36,37}$.

\section{CONCLUSÃO}

A inflamação crônica, característica da obesidade, resulta da liberação de adipocinas pró-inflamatórias pelos adipócitos, que atraem macrófagos para área afetada, desencadeando o processo inflamatório e o estresse oxidativo. Dessa forma, destacam-se os alimentos com propriedades funcionais que atuam na modulação da inflamação e do estresse oxidativo. A inserção desses alimentos que apresentam, em sua composição, compostos bioativos, como polifenóis, catequinas 
4 Obesidade: inflamação e compostos bioativos

e flavonoides, contribui para a homeostase e o funcionamento normal do organismo.

Entre os alimentos, destacam-se açafrão, chá-verde, romã, aveia e azeite de oliva como alimentos importantes na prevenção e no tratamento da obesidade, tendo em vista o potencial dos alimentos em modular a inflamação na obesidade, o conhecimento de suas propriedades funcionais é importante por auxiliar os profissionais de Nutrição no planejamento de estratégias para a prevenção e o tratamento da obesidade.

\section{REFERENCES}

1. Dahiya DK, Renuka, Puniya M, Shandilya UK, Dhewa T, Kumar N. Gut Microbiota Modulation and Its Relationship with Obesity Using Prebiotic Fibers and Probiotics: A Review. Front Microbiol. 2017 Apr; 8(563):1-17. doi: 10.3389/ fmicb.2017.00563doi:10.3389/fmicb.2017.00563.

2. Ministério da Saúde (BR). Secretaria de Vigilância em Saúde. Vigitel Brasil: vigilância de fatores de risco e proteção para doenças crônicas por inquérito telefônico: estimativas sobre frequência e distribuição sociodemográfica de fatores de risco e proteção para doenças crônicas nas capitais dos 26 estados brasileiros e no Distrito Federal. Brasília: Ministério da Saúde; 2018.

3. Crawford M, Whisner C, Al-Nakkash L, Sweazea KL. Six-Week High-Fat Diet Alters the Gut Microbiome and Promotes Cecal Inflammation, Endotoxin Production, and Simple Steatosis without Obesity in Male Rats. Lipids. 2019 Feb; 54(2-3):119-131. doi:10.1002/lipd.12131.

4. Yang B-G, Hur KY, Lee M-S. Alterations in Gut Microbiota and Immunity by Dietary Fat. Yonsei Med J. 2017 Nov; 58(6):1083. doi:10.3349/ ymj.2017.58.6.1083.

5. Milenkovic D, Morand C, Cassidy A, Konic-Ristic A, Tomás-Barberán F, Ordovas $\mathrm{JM}$, et al. Interindividual Variability in Biomarkers of Cardiometabolic Health after Consumption of Major Plant-Food Bioactive Compounds and the Determinants Involved. Adv Nutr. 2017 Jul; 8(4):558-570. doi:10.3945/an.116.013623.

6. Dias PC, Henriques P, Anjos LA dos, Burlandy L. Obesidade e políticas públicas: concepções e estratégias adotadas pelo governo brasileiro. Cad Saude Publica. 2017; 33(7):1-12. doi:10.1590/0102-311x00006016

7. Ellulu MS, Patimah I, Khaza'ai H, Rahmat A, Abed Y. Obesity and inflammation: the linking mechanism and the complications. Arch Med Sci. 2017 Jun; 4(13): 851-863. doi:10.5114/aoms.2016.58928.

8. Fernández-Sánchez A, Madrigal-Santillán E, Bautista $M$, Esquivel-Soto J, Morales-Gonzalez A, Esquivel-Chirino C, et al. Inflammation, Oxidative Stress, and Obesity. Int J Mol Sci. 2011 May;12(5):3117-3132. doi:10.3390/ ijms12053117.

9. Crujeiras AB, Carreira MC, Cabia B, Andrade S, Amil M, Casanueva FF. Leptin resistance in obesity: An epigenetic landscape. Life Sci. 2015 Nov; 140(1): 57-63. doi:10.1016/j.Ifs.2015.05.003.

10. Abizaid A. Stress and obesity: The ghrelin connection. J Neuroendocrinol. 2019 Jul; 31(7):1-8. doi:10.1111/jne.12693.

11. Makris MC, Alexandrou A, Papatsoutsos EG, Malietzis G, Tsilimigras DI, Guerron AD, et al. Ghrelin and Obesity: Identifying Gaps and Dispelling Myths. A Reappraisal. In Vivo (Brooklyn). 2017 Nov-Dec; 31(6):1047-1050. doi:10.21873/ invivo.11168.

12. Monteiro R, Azevedo I. Chronic Inflammation in Obesity and the Metabolic Syndrome. Mediators Inflamm. 2010; 2010:1-10. doi:10.1155/2010/289645.

13. Saltiel AR, Olefsky JM. Inflammatory mechanisms linking obesity and metabolic disease. J Clin Invest. 2017 Jan; 127(1):1-4. doi:10.1172/JCI92035.

14. Balsan GA, Vieira JL da C, Oliveira AM de, Portal VL. Relationship between adiponectin, obesity and insulin resistance. Rev Assoc Med Bras. 2015 Jan-Feb; 61(1):72-80. doi:10.1590/1806-9282.61.01.072.

15. Rodríguez-Hernández H, Simental-Mendía LE, Rodríguez-Ramírez G,
Reyes-Romero MA. Obesity and Inflammation: Epidemiology, Risk Factors, and Markers of Inflammation. Int J Endocrinol. 2013 Apr; 2013(1):1-11. doi:10.1155/2013/678159.

16. Teodoro AJ. Bioactive Compounds of Food: Their Role in the Prevention and Treatment of Diseases. Oxid Med Cell Longev. 2019; 2019(1):1-4 doi:10.1155/2019/3765986.

17. Serrano J, Cassanye A, Martín-Gari M, Granado-Serrano A, Portero-Otín M. Effect of Dietary Bioactive Compounds on Mitochondrial and Metabolic Flexibility. Diseases. 2016 Mar; 4(1):14. doi:10.3390/diseases4010014.

18. Miyazawa T, Nakagawa K, Kim SH, Thomas MJ, Paul L, Zingg J-M, et al. Curcumin and piperine supplementation of obese mice under caloric restriction modulates body fat and interleukin-1ß. Nutr Metab (Lond). 2018;15(1):12. doi:10.1186/s12986-018-0250-6.

19. Shehzad A, Ha T, Subhan F, Lee YS. New mechanisms and the antiinflammatory role of curcumin in obesity and obesity-related metabolic diseases. Eur J Nutr. 2011 Apr; 50(3):151-161. doi:10.1007/s00394-011-0188-1.

20. Sahebkar A, Mohammadi A, Atabati A, Rahiman S, Tavallaie S, Iranshahi $M$, et al. Curcuminoids Modulate Pro-Oxidant-Antioxidant Balance but not the Immune Response to Heat Shock Protein 27 and Oxidized LDL in Obese Individuals. Phyther Res. 2013;27(12):1883-1888. doi:10.1002/ptr.4952.

21. Ganjali S, Sahebkar A, Mahdipour E, Jamialahmadi K, Torabi S, Akhlaghi $S$, et al. Investigation of the Effects of Curcumin on Serum Cytokines in Obese Individuals: A Randomized Controlled Trial. Sci World J. 2014; 2014(1):1-6. doi:10.1155/2014/898361.

22. Nabi BN, Sedighinejad A, Haghighi M, Fazi F, Rimaz S, Atrkarroushan, et al. The Anti-Obesity Effects of Green Tea: A Controlled, Randomized, Clinical Trial. Iran Red Crescent Med J. 2018;20(1):1-7. doi:10.5812/ircmj.55950.

23. Tran J. Green Tea: A Potential Alternative Anti-Infectious Agent Catechins and Viral Infections. Adv Anthropol. 2013; 03(04):198-202. doi:10.4236/ aa.2013.34028.

24. Rains TM, Agarwal S, Maki KC. Antiobesity effects of green tea catechins: a mechanistic review. J Nutr Biochem. 2011 Jan; 22(1):1-7. doi:10.1016/j. jnutbio.2010.06.006

25. Huang J, Wang Y, Xie Z, Zhou Y, Zhang Y, Wan X. The anti-obesity effects of green tea in human intervention and basic molecular studies. Eur J Clin Nutr. 2014 Oct; 68(10):1075-1087. doi:10.1038/ejcn.2014.143.

26. Reygaert W. An Update on the Health Benefits of Green Tea. Beverages. 2017; 3(4):6. doi:10.3390/beverages3010006

27. Al-Muammar MN, Khan F. Obesity: The preventive role of the pomegranate (Punica granatum). Nutrition. 2012 Jun; 28(6):595-604. doi:10.1016/j. nut.2011.11.013.

28. Ammar AE, Esmat A, Hassona MDH, Tadros MG, Abdel-Naim AB, Guns EST. The effect of pomegranate fruit extract on testosterone-induced $\mathrm{BPH}$ in rats. Prostate. 2015 May; 75(7):679-692. doi:10.1002/pros.22951.

29. Kim YE, Hwang CJ, Lee HP, Kim CS2, Son DJ1, Ham YW3, et al. Inhibitory effect of punicalagin on lipopolysaccharide-induced neuroinflammation, oxidative stress and memory impairment via inhibition of nuclear factor- 
kappaB. Neuropharmacology. 2017 May; 117(1):21-32. doi:10.1016/j. neuropharm.2017.01.025.

30. Danesi F, Ferguson L. Could Pomegranate Juice Help in the Control of Inflammatory Diseases? Nutrients. 2017 Aug; 9(9):958. doi:10.3390/ nu9090958.

31. Chang HC, Huang CN, Yeh DM, Wang SJ, Peng CH, Wang CJ. Oat Prevents Obesity and Abdominal Fat Distribution, and Improves Liver Function in Humans. Plant Foods Hum Nutr. 2013 Mar; 68(1): 18-23. doi:10.1007/s11130013-0336-2.

32. Gill PA, van Zelm MC, Muir JG, Gibson PR. Review article: short chain fatty acids as potential therapeutic agents in human gastrointestinal and inflammatory disorders. Aliment Pharmacol Ther. 2018 Jul; 48(1):15-34. doi:10.1111/apt.14689.

33. Parada Venegas D, De la Fuente MK, Landskron G, González MJ, Quera R, Dijkstra G, et al. Short Chain Fatty Acids (SCFAs)-Mediated Gut Epithelial and Immune Regulation and Its Relevance for Inflammatory Bowel Diseases. Front
Immunol. 2019 Mar;10:277. doi:10.3389/fimmu.2019.00277.

34. Bozzetto L, Costabile G, Della Pepa G, Ciciola P, Vetrani C, Vitale M, et al. Dietary Fibre as a Unifying Remedy for the Whole Spectrum of ObesityAssociated Cardiovascular Risk. Nutrients. 2018 Jul;10(7): E943. doi:10.3390/ nu10070943.

35. Souza P, Marcadenti A, Portal V. Effects of Olive Oil Phenolic Compounds on Inflammation in the Prevention and Treatment of Coronary Artery Disease. Nutrients. 2017 Sep; 9(10):1087. doi:10.3390/nu9101087.

36. Santangelo C, Vari R, Scazzocchio B, De Sanctis P, Giovannini C, D'Archivio $M$, et al. Anti-inflammatory Activity of Extra Virgin Olive Oil Polyphenols: Which Role in the Prevention and Treatment of Immune-Mediated Inflammatory Diseases? Endocr Metab Immune Disord Drug Targets. 2018 ;18(1):36-50. doi:1 $0.2174 / 1871530317666171114114321$.

37. Estruch R, Ros E, Salas-Salvadó J, Cova MI,Corella D,Arós F, et al. Primary Prevention of Cardiovascular Disease with a Mediterranean Diet. N Engl J Med. 2013 Apr; 368(14):1279-1290. doi:10.1056/NEJMoa1200303. 